\title{
Virtuoses GitarRenspiel im RoCK Und METAL. ZUM EINFLUSS VON VERZERRUNG AUF DAS »SHREDDING «
}

\begin{abstract}
Jan-Peter Herbst
Als Sinnbild für den spieltechnisch herausragenden Instrumentalvirtuosen wird gemeinhin Nicolò Paganini angesehen (vgl. Metzner 1998: 113ff.). Er setzte nicht nur performativ neue Maßstäbe, sondern lieferte mit dem Klang und der Spielweise seiner Violine eine perfekte Vorlage für das Gitarrenspiel in der Rockmusik in den 1960er und 1970er Jahren (vgl. Middleton 1990: 30ff.; Walser 1993: 63; Custodis 2011: 1). Es dürfte also kaum dem Zufall geschuldet sein, dass vor allem frühe Rock- und Metal-Gitarristen wie Ritchie Blackmore und Uli Jon Roth gleichzeitig die Grenzen verzerrten Gitarrenspiels ausloteten und barocke wie auch klassische Melodie- und Motivtechniken adaptierten (vgl. Middleton 1990: 30ff., Walser 1993: 63ff.). Nachfolgende Spieler wie Randy Rhoads und Yngwie Malmsteen entwickelten diesen Ansatz weiter und nutzten die Instrumententechnologie, um spieltechnische Schwierigkeiten zu überwinden. Obwohl Rhoads um 1980 auf ein größeres Verzerrungspotential als seine Vorbilder zurückgreifen konnte, nahm er seine Soli stets auf drei Spuren auf, um durch den zwar weniger transparenten aber volleren Sound den Eindruck eines flüssigeren Spiels zu erzielen (vgl. Obrecht 2010). Malmsteen dagegen höhlte in der Tradition Blackmores sein Gitarrengriffbrett aus, um dank des geringeren Fingerkontakts kraftsparender spielen zu können (vgl. Frudua 2010). Diese Entwicklung kulminierte schließlich im »Shred-Style« des Hair und Heavy Metal der 1980er Jahre (vgl. Walser 1993: 101).

Auch wenn das effektvolle Solospiel in den Charts ab den 1990er Jahren durch den Erfolg des Grunge ein jähes Ende fand (vgl. Waksman 2003b: 128 ), spielt es in vielen Stilrichtungen des Rock und Metal nach wie vor eine bedeutende Rolle, wie gitarrenorientierte Literatur (vgl. Waksman 2003b: 124ff.), Stilanalysen (vgl. Elflein 2010: 305ff.) und auch journalistische Auflistungen der vermeintlich besten Gitarristen (vgl. Mclver 2008: 9) demonstrieren. Noch deutlicher weist Custodis darauf hin, dass Rock neben dem
\end{abstract}


Jazz eines der wenigen Genres sei, in denen technische Virtuosität nicht nur geschätzt, sondern zum Teil sogar eingefordert werde:

»most clichés and connotations of the guitar virtuoso combine elements of historic role models of 19th century's icons Paganini and Liszt with the distortion sound and the habitus of playing an electric guitar« (Custodis 2011: 1).

Der Zusammenhang von Gitarrenverzerrung ${ }^{1}$ und schnellem Solospiel wurde bislang nur vermutet (vgl. Walser 1993: 57ff.), ein akustischer Nachweis aber nicht erbracht. Hinweise darauf, dass Rocksoli während der letzten fünfzig Jahre schneller wurden, liefern Slaven/Krout (2016) mit einer empirischen Studie, die jedoch keine Erklärungen bereitstellt. Weitere Studien beschäftigen sich mit virtuosem E-Gitarrenspiel oder mit spezifischen Spielweisen, ohne explizit die Verzerrung zu berücksichtigen (vgl. Waksman 2001: 128f., 2003b: 124ff.). Experimentelle Untersuchungen existieren nur wenige und auch die detaillierteste Sound-Studie von Einbrodt (1997) thematisiert nicht den Zusammenhang von Verzerrung und schneller Spieltechnik, sodass ein populärmusikwissenschaftlicher Nachweis noch aussteht. Abgesehen von diesem Desiderat spielt das Thema Verzerrung auch für Gitarristen eine große Rolle, wie vor allem Diskussionen in Online-Foren zeigen. Dort wird der Gebrauch von Verzerrung mit Bezug zur spieltechnischen »Leistung « - meist im Sinne eines schnellen Spiels unter Anwendung diverser Spieltechniken wie Wechselschlag, Sweeping, Hammer-On, Pull-Off oder Zweihand-Tapping - kritisch abgewogen, und gleichsam werden Übestrategien für künftige »Shredder « ausgetauscht.

Der vorliegende Beitrag geht deshalb den Fragen nach, welche Folgen starke Verzerrung für ein virtuoses Solospiel im Rock und Metal hat. Die Studie beginnt mit einer Inhaltsanalyse von Argumentationen aus OnlineForen. Die daran anschließende Analyse der Verzerrung untersucht allgemeine Klangcharakteristika und ihre Auswirkungen auf die Grundtechniken des Instruments. Als experimenteller Ansatz beruht die Analyse auf zwei Fallstudien aus der Rockgeschichte. Abschließend erfolgt die Reflexion der analytisch-empirischen Ergebnisse zum verzerrten Spiel.

1 Für eine detaillierte Analyse von Gitarrenverzerrung siehe Herbst 2016: 35-42, $115-142$. 


\section{Gitarrenverzerrung in Online-Musikerforen}

Per Google-Suche wurden mit den Stichworten »Verzerrung (bzw. »distortion $\ll$ ) und »Gitarre $\ll$ (bzW. »guitar $\ll$ ) relevante Postings in Musiker- und Gitarrenforen ausfindig gemacht. Weitere Suchvorgänge innerhalb der entsprechenden Foren ergänzten die Google-Suche. Durch manuelle Auswahl wurden Themen herausgefiltert, die nicht das Gitarrenspiel bzw. das »Shredding « betrafen. Neben dem Musiker-Board als größtem deutschsprachigen Forum fanden das Shred Academy Board, das Ultimate Guitar Board und das Guitar Masterclass Board Eingang in die Analyse. Eine derartige Auswahl ließ zwar keine repräsentative Auswertung zu, aber sie lieferte einen bedeutsamen Einblick in die diskutierten Themen. Um die Auswertung zu strukturieren, wurde eine zusammenfassende qualitative Inhaltsanalyse mithilfe von MAXQDA 10 durchgeführt (vgl. Kuckartz 2010). Untersucht wurden 21 zum Teil mehrseitige Threads, deren Überschriften sich einteilen lassen in 1. Spieltechniken (Wechselschlag, Sweeping, Legato, Tapping), 2. "Shredding « und 3. »Cheating «. Die Detailanalyse machte allerdings schnell deutlich, dass in allen Threads die angeblich spielvereinfachende oder gar »betrügerische « Auswirkung der Verzerrung einigen Komplikationen gegenübersteht. Implizit ist stets ein Wettbewerbsgedanke erkennbar, wie er als charakteristischer Bestandteil von Virtuosität beschrieben wird (vgl. Pincherle 1949: 227).

Die Themenüberschriften deuten bereits an, dass das Thema »Cheating « ausgiebig diskutiert wird - vor allem bezüglich der Soundgestaltung. Die Inhaltsanalyse bestätigt diese Annahme, denn sie zeigt eine Fülle an Maßnahmen zur Instrumentenpräparation, um das Spiel in einem besseren Licht erscheinen zu lassen. Genannt werden die Verwendung von dünneren Saiten für einen geringeren Kraftaufwand, ein mehrfaches Aufnehmen von Soli für eine flüssigere Wirkung, alternative Stimmungen sowie die Nutzung von aktiven Tonabnehmern mit mehr Ausgangsleistung. Ferner werden die Effekte Wah Wah, Echo und Hall als Mittel für das Verschleiern von Spielfehlern beschrieben. Die Frequenzbearbeitung spielt ebenfalls eine Rolle, da durch den dumpferen Hals-Tonabnehmer oder den Ton-Potentiometer die Transparenz reduziert und dadurch ein sauberes Spiel »vorgegaukelt « werden könne. In ähnlicher Weise wird ausgiebig die Verwendung eines Haarbandes diskutiert, das durch ein sanftes Aufliegen auf den Saiten ungewünschte Saitenschwingungen verhindern und somit Nebengeräusche herausfiltern könne. Auf spieltechnischer Ebene stellt sich die Frage, ob der Verzicht auf 
einen regelmäßigen Anschlag zugunsten einer Legato-Spielweise als Betrug zu werten sei.

Der quantitativ bedeutendste Kritikpunkt betrifft die Verzerrung, weil sie potenziell unsauberes oder fehlerhaftes Spiel maskieren könne. So äußert Nutzer Jak888 im Shred Academy Board: »when you get a Boss MT-2 (metalzone), that distortion, overdrive and compression thing, all at once, you can shred like a god, just because the sound hides all your mistakes. « Ähnlich repräsentativ ist die Aussage von Maidenheadsteve im Ultimate Guitar Board: "The closest thing I can think of [bezüglich >cheating $<$ is hiding behind lots of distortion so it sounds unclear to cover up technical deficiency. «One Vision resümiert im Ultimate Guitar Board daher bezüglich des Verzerrungsgrads: „To a true virtuoso, the amount of gain doesnt matter, he or she can play it either way.«

Neben der ethischen Dimension des Betrugs existieren Aussagen, die Verzerrung gar als notwendige Voraussetzung für einige Spieltechniken erkennen lassen. Bspw. äußert der bereits zitierte One Vision:

»I still feel cheap when I use lots of it. But, like when I really need it for something like diminished LEGATO string skipping. Come on. Mad legato stretches. I tried doing it with less gain and I can't get the Tap-Slides to sound AT ALL.«

Abgesehen von der Kritik an den potenziell spielvereinfachenden Auswirkungen der Verzerrung, gibt es eine vernachlässigbare kleine Anzahl von Aussagen, welche die eingeschränkte Artikulation des verzerrten Stils betonen; zum Beispiel Vercingetorix im Shred Academy Board:

»Now one of the good reasons to practice clean is so you can hear what you are playing with nothing hiding your mistakes, listening for the clean distinction between each note, even timing for each pick strike, and that they are all coming in at the same volume. All these characteristics can be masked by playing with gain and FX (reverb, delay, phaser, compressor and wah) you get the drift.«

Dieser Kritik stehen einige wenige Äußerungen gegenüber, die die Herausforderungen beim Spiel mit Verzerrung hervorheben, etwa die Schwierigkeit, mit stark verzerrtem Sound noch dynamisch differenziert zu spielen. Ein weiterer Nutzer argumentiert, dass Verzerrung Spielfehler noch offensichtlicher mache. Andere heben hauptsächlich die schwer kontrollierbaren kompressionsbedingten Nebengeräusche hervor. Bspw. schreibt Matrix Claw im Ultimate Guitar Board:

»I feel the complete opposite. Playing clean won't show any of your lack of muting strings and playing cleanly, I don't understand why people say that at 
all. If anything, playing clean hides sloppiness because it's not being amplified as distortion and is often times in-audible when playing on a clean channel. I know I sound a hell of a lot better playing shred on the clean channel than I do on the distortion channel «. ${ }^{2}$

Insbesondere im Zusammenhang mit den Spieltechniken Sweeping und Tapping wird diese Notwendigkeit betont.

Wenngleich in den Diskussionen die Kritik an den täuschenden Maßnahmen und insbesondere an der Verzerrung weit verbreitet ist, legt eine Umfrage im Ultimate Guitar Forum ${ }^{3}$ über den Verzerrungsgrad beim "Shredding « doch nahe, dass die meisten Spieler nicht auf die positiven Effekte verzichten möchten. Während von den 76 Befragten nur $5 \%$ angeben, keine Verzerrung zu nutzen und weitere $18 \%$ nur wenig »Gain«, so spielt die Mehrheit nach eigenen Aussagen mit viel (47\%) oder extrem viel $(29 \%)$ Verzerrung. Diese Angaben können als Indiz für die wettbewerbsorientierte Denkweise gedeutet werden, weil ein geringerer Verzerrungsgrad potenziell einem spielerischen Nachteil gleichkommen könnte. Wenn auch die wenigsten Nutzer solcher Online-Boards auf einer Bühne live gegeneinander »antreten «, gibt es doch eine rege Community in Foren und auf Videoplattformen, wo Cover von spieltechnisch anspruchsvollen Stücken vorgestellt werden, deren Spieler der Kritik ausgesetzt sind. ${ }^{4}$ Daneben existieren in Videoplattformen unzählige »Wettkämpfe« bekannter »Shred«-Gitarristen wie Michael Angelo Batio, Rusty Cooley, Paul Gilbert, Marty Friedman oder Joe Satriani, die sich entweder durch zusammengestellte Kompilationen virtuell oder in speziellen Shows live »duellieren«. Ein weiteres Phänomen sind Reihen wie »Betcha Can't Play This!«, in denen renommierte Gitarristen ihre anspruchsvollsten »Licks« vorstellen und damit Amateure zum Wetteifern anspornen.

2 »How To Set Amp/Effects For Shred«, verfügbar unter: https://www.ultimateguitar.com/forum/showthread.php?t=987527 (Stand vom 23.3.2017).

3 »How many of you shred with distortion? «, verfügbar unter: https://www. ultimate-guitar.com/forum/showthread.php? $\mathrm{t}=802738$ (Stand vom 23.3.2017).

4 Vgl. bspw. die Themen »An alle fortgeschrittenen Gitarristen: Zeigt[,] was ihr könnt« oder »Zeigt[,] was ihr könnt!: Metal Style« im Musiker-Board. 


\section{Analyse der Auswirkung von Verzerrung auf das Spiel}

\section{Methode}

Die Analyse des Einflusses von Soundcharakteristika auf die Spiel- und Ausdrucksfähigkeit profitiert von einem multimethodischen Ansatz. Strukturorientierte Methoden können klangliche Phänomene kaum adäquat erfassen (vgl. Schneider 2002: 111), weshalb akustische, strukturelle und perzeptive Verfahren kombiniert wurden. In einem ersten Schritt fand die Visualisierung der akustischen Eigenschaften durch detaillierte Spektraldarstellungen (Sonic Visualiser) und Wellenformendiagramme (Audacity) statt. Hiermit konnte einerseits ein besseres Verständnis über die gegenseitige Beeinflussung spektraler und dynamischer Eigenschaften gewonnen werden. Andererseits trugen die Abbildungen als objektive Repräsentationen zur Erfüllung empirischer Qualitätskriterien wie Wiederholbarkeit und Transparenz bei (vgl. Cook/Clarke 2004: 4). Zusätzlich wurden einzelne Töne für eine akustische Feature-Analyse exportiert, um spezifische Eigenschaften verschiedener Spieltechniken zu identifizieren. Die Features wurden mit der MIR Toolbox (vgl. Lartillot/Toiviainen 2007) extrahiert. Das Dynamikverhalten wurde außerdem mit Adobe Audition 3 überprüft. Zur methodischen Absicherung und für einen tiefergehenden Erkenntnisgewinn wurden die unterschiedlichen visuellen und statistischen Ergebnisse trianguliert.

In dem experimentellen Ansatz dienten eigens aufgenommene Ausschnitte veröffentlichter Rock- und Metalsoli als Analysematerial. Die Sampleauswahl unterlag weniger der musikhistorischen Bedeutung als dem Einsatz wesentlicher Spieltechniken innerhalb eines kurzen Ausschnittes. »Groove Or Die« (1997) des Gitarrenvirtuosen Andy Timmons wurde nicht nur wegen der hohen spieltechnischen Herausforderungen gewählt, sondern auch weil mehrere Anschlagstechniken in einer Phrase verglichen werden konnten. Für die Legato-Technik wurde aufgrund der schnellen Spielweise das Intro-Solo von Queens »I Want It All« (1989) gewählt.

Um die akustischen Effekte der Gitarrenverzerrung analysieren zu können, wurden Aufnahmen mit verschiedenen Sounds erstellt. Gegenüber einer Analyse originaler Hörbeispiele konnte mit dem experimentellen Vorgehen eine weit bessere Kontrolle über relevante Variablen, insbesondere Verzerrung, ausgeübt werden. Des Weiteren konnten so Interferenzen mit anderen Instrumenten ausgeschlossen werden (vgl. Einbrodt 1997: 18ff.). Jede Performance wurde zunächst über eine DI-Box direkt in den Sequenzer 
(Apple Logic Pro 9 mit Presonus FirePod Soundkarte) eingespielt. Mit der Palmer Daccapo Box konnten die Signale mehrfach mit verschiedenen Verstärkereinstellungen aufgenommen werden (»Re-Amping«). Drei Sounds wurden im Crunch-Kanal eines Marshall JCM 2000 TSL Röhrenverstärkers erzeugt: Ein unverzerrter Sound mit niedriger »Gain«-Einstellung (Clean), ein mittelmäßig verzerrter Sound (Overdrive) und ein stark verzerrter Sound (Distortion), für den ein Fulltone OCD Pedal hinzugefügt wurde. Als Box wurde das Modell 1960BV von Marshall mit Celestion G12 Vintage 30 Lautsprechern genutzt. Mikrofoniert wurden die Aufnahmen mit einem dynamischen Shure SM57 nah vor dem Lautsprecher. Insgesamt repräsentierte die Verstärkungsanlage ein weitverbreitetes Setup in Rock- und Metal-Genres seit den 1970er Jahren. Transistor- und moderne Simulationsverstärker wurden wegen der nach wie vor geringen Verbreitung (vgl. Herbst 2016: 300f.) nicht berücksichtigt. Mit einer ähnlich traditionsbewussten Zielsetzung wurden auch die Gitarren ausgewählt: Eine Fender American Stratocaster mit Seymour Duncan SH-4 Humbucker am Steg für »Groove Or Die« und eine Gibson Les Paul mit 490T Tonabnehmer für »| Want It All«. Diese Gitarren sind in den genannten Genres immer noch beliebt (vgl. ebd.: 298f.). Beim Audio-Export wurden die Produktionen zwecks vergleichbarer Lautheit auf -0.1 dBFS normalisiert.

\section{Allgemeine Klangcharakteristika}

Um die akustischen Veränderungen der Verzerrung als Vorbereitung für die Fallanalysen grundlegend zu betrachten, wurde der Einzelton E5 $(659 \mathrm{~Hz})$ visuellen und quantitativen Verfahren unterzogen. Eine erste visuelle Annäherung erfolgte anhand von Spektral- und Wellenformdarstellungen.

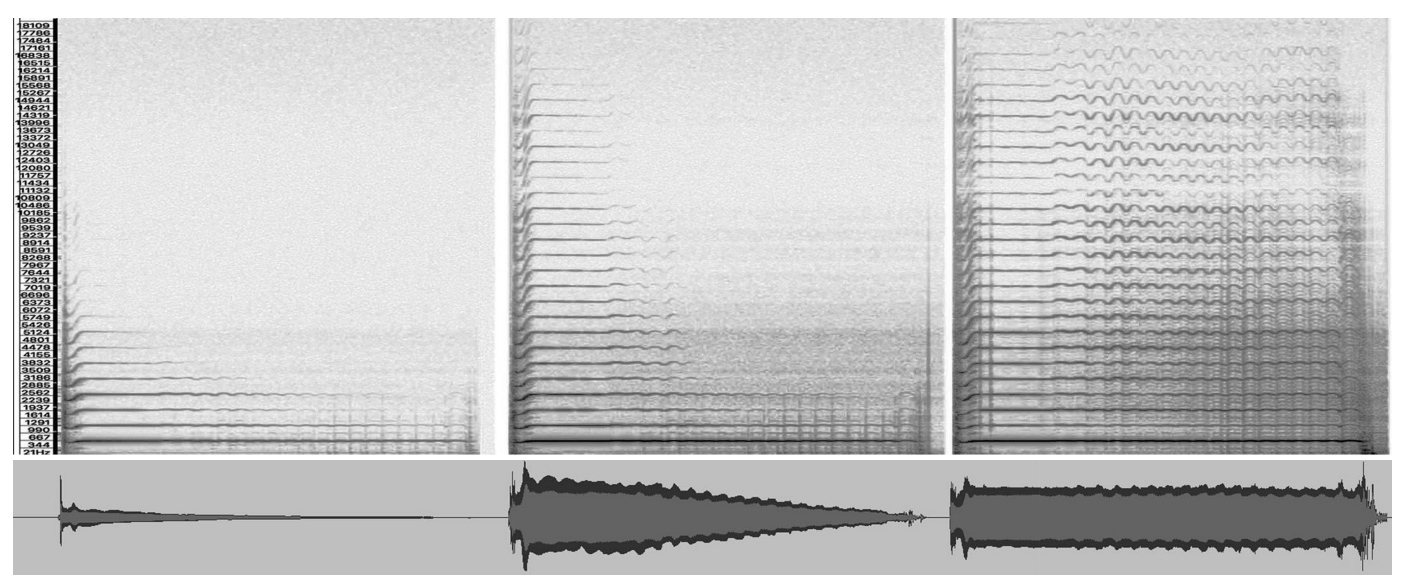

Abbildung 1: Ton einer Gibson Les Paul mit Vibrato und Saitenziehen.

Links: Clean, Mitte: Overdrive, rechts: Distortion; 21 bis $18.000 \mathrm{~Hz}$ 
Die Gitarre produziert, wie das Spektrogramm veranschaulicht, periodische Wellen. Die tiefste Schwingung ist in diesem Fall der Grundton, die folgenden sind harmonische Obertöne. Zur Klangcharakteristik des Instruments tragen die Anzahl der Teiltöne, ihr Lautstärkenverhältnis und ihre zeitliche Entwicklung wesentlich bei (vgl. Pierce 1985). Bei der unverzerrten Gitarre lassen sich im Spektrogramm neben der Grundschwingung drei Obertöne erkennen, die anzeigen, dass der primäre Frequenzbereich zwei Oktaven beträgt. Die moderat verzerrte Aufnahme unterscheidet sich von der unverzerrten nur geringfügig. Nach wie vor sind die ersten vier Teiltöne dominant, wobei auch höherrangige Obertöne zu erkennen sind, die allerdings kurz nach dem Anschlag verklingen. Dagegen unterscheidet sich die sehr verzerrte Gitarre deutlich, denn sämtliche harmonischen Obertöne sind verstärkt und erweitern das Signal auf einen Frequenzbereich von fast fünf Oktaven. Trotz des eingeschränkten Reproduktionsraums des Lautsprechers bis ca. $5 \mathrm{kHz}$, der in den Spektrogrammen an dem Geräuschband klar zu erkennen ist, sind Klanganteile von beträchtlicher Lautstärke im Höhen- und Präsenzbereich vorhanden. So verfügen höhere Obertöne wie etwa A9 $(13.290 \mathrm{~Hz})$ mit $-35 \mathrm{~dB}$ gegenüber dem ersten Oberton E6 $(1.319 \mathrm{~Hz})$ mit -25 dB über eine nur wenig geringere Lautheit. Der Vergleich der drei Sounds in der dreidimensionalen Darstellung verdeutlicht folglich, dass der in der Wellenform erkennbare Kompressionseffekt nicht linear verläuft, sondern in Abhängigkeit zur Frequenz steht. Ein geringes Hinzufügen von Verzerrung intensiviert vor allem die unteren Teiltöne im Bass- und Mittenregister. Deutlich mehr Verzerrung scheint notwendig, um auch die höheren Teiltöne zu verstärken und über einen längeren Zeitraum schwingen zu lassen. Dieses als »Sustain« bezeichnete Merkmal erweitert die Möglichkeiten der E-Gitarre als Melodieinstrument erheblich (vgl. Middleton 1990: 30ff.; Walser 1993: 63ff.; Herbst 2016: 143-237). Neben diesen frequenzabhängigen Kompressionseffekten veranschaulicht die Spektralanalyse, dass hauptsächlich harmonische Teiltöne (vgl. Pierce 1985: 37f.) und weniger ganze Frequenzbereiche verstärkt werden. Andernfalls würde der Sound an Klarheit verlieren und eine durchdringende Schärfe erhalten (vgl. Doyle 1993: 56f.).

Da die Studie davon ausging, dass selbst geringe akustische Veränderungen beträchtlichen Einfluss auf die Spielfähigkeit nehmen, wurden die Aufnahmen einer Feature-Analyse unterzogen: 


\begin{tabular}{l|l|l|l} 
& Clean & Overdrive & Distortion \\
\hline Primärer Frequenz- & E5 $(659 \mathrm{~Hz})-$ & E5 $(659 \mathrm{~Hz})-$ & E5 $(659 \mathrm{~Hz})-$ \\
bereich & E7 $(2.637 \mathrm{~Hz})$ & E7 $(2.637 \mathrm{~Hz})$ & D10 $(18.323 \mathrm{~Hz})$ \\
Anschlagszeit & $32 \mathrm{~ms}$ & $48 \mathrm{~ms}$ & $50 \mathrm{~ms}$ \\
Anschlagsphase & $0,5 \mathrm{~s}$ & $1,77 \mathrm{~s}$ & $3,53 \mathrm{~s}$ \\
Lautheit (RMS) & $-27,86 \mathrm{~dB}$ & $-11,55 \mathrm{~dB}$ & $-6,77 \mathrm{~dB}$ \\
Spektraler Mittelwert & $1.855 \mathrm{~Hz}$ & $1.321 \mathrm{~Hz}$ & $2.118 \mathrm{~Hz}$
\end{tabular}

Tabelle 1: Akustische Features der drei Sounds

Im Fokus standen vor allem Aspekte der Hüllkurve, die sich spektralanalytisch nur grob erfassen lassen. Während allgemein viele Instrumente in der »Sustain«-Phase ähnlich klingen, nimmt die Anschlagsphase großen Einfluss auf den Instrumentenklang (vgl. Barkowsky 2009: 71). Eine »Gain«-Zunahme von einem unverzerrten zu einem moderat verzerrten Gitarrensound erhöht die Anschlagszeit, d.h. die Zeit vom Saitenkontakt bis zur maximalen Ausschwingung (vgl. Lartillot/Toiviainen 2007: 114), um circa ein Drittel. Mehr Verzerrung verlängert die Anschlagszeit kaum weiter. Dagegen reagiert die Anschlagsphase als die Zeit zwischen dem Anschlag und der Ausschwingphase (vgl. ebd.: 122) sensibel auf den Verzerrungsgrad, sodass sich die Phase von Clean zu Overdrive ungefähr verdreifacht und von Overdrive zu Distortion nochmal verdoppelt. Die festgestellten Veränderungen in der Hüllkurve lassen den Schluss zu, dass Verzerrung die Definition des Anschlags sowohl aufgrund unpräziserer Anschlagsphasen und -zeiten als auch wegen der größeren Geräuschhaftigkeit des Plektrumanschlags negativ beeinflusst. Letzteres ist im Spektrogramm ersichtlich.

Die Dynamik unterliegt erwartungsgemäß größeren Veränderungen durch den Verzerrungsgrad. Die durchschnittliche Lautheit (RMS in dBFS) erhöht sich von Clean zu Overdrive um $141 \%$ und von Overdrive zu Distortion um weitere $71 \%$. Die Helligkeit, berechnet aus dem spektralen Mittelwert (vgl. McAdams/Depalle/Clarke 2004: 191), verändert sich ebenfalls je nach Verzerrungsgrad (Tab. 1). Ein moderates Hinzufügen von Verzerrung senkt den spektralen Mittelwert beträchtlich. Die Spektraldarstellung verdeutlicht, dass nichtlineare Verzerrung nicht nur die Obertöne verstärkt, sondern auch die Lautstärkerelation der Teiltöne beeinflusst. Im vorliegenden Fall wird die Grundtonschwingung um ca. 6 dB lauter, was ungefähr einer doppelten Lautstärke entspricht (vgl. Barkowsky 2009: 31). Dadurch werden die leicht verstärkten Obertöne ausgeglichen und der Ton bekommt einen wärmeren, bassbetonteren Sound. Anders verhält es sich bei der Distortion-Aufnahme, wo die starke Verzerrung die Grundtonfrequenz zwar ebenfalls deutlich an- 
hebt, allerdings in den oberen Harmonischen verhältnismäßig lauter und langanhaltender. Dies führt zu einer Erhöhung des spektralen Mittelwerts und somit der Helligkeit. Ein solch heller Sound könnte sehr verzerrten Gitarren mehr Klarheit verleihen und die negativen Effekte der undefinierteren Hüllkurvenentwicklung teilweise ausgleichen.

\section{Spiel mit Plektrum}

Die Tonerzeugung auf der elektrischen Gitarre erfolgt im Normalfall mit einem Plektrum. Wie Einbrodt (1997) in seiner experimentellen Untersuchung detailliert nachwies, ist die Art des Anschlags für den Gitarrensound von entscheidender Bedeutung. Inwiefern sich die Verzerrung auf das Spiel mit dem Plektrum auswirkt, soll exemplarisch an »Groove Or Die« (1997) untersucht werden:

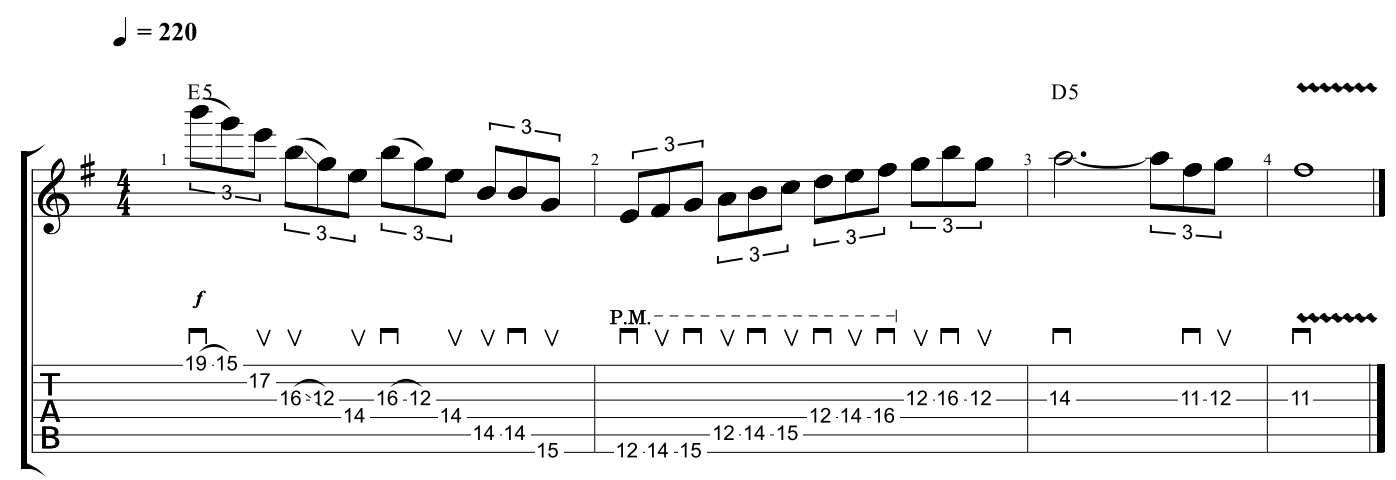

Abbildung 2: Anfangsmotiv von Andy Timmons' »Groove Or Die« (1997)

Das Arpeggio im ersten Takt des Anfangsmotivs stellt für das Plektrumspiel eine Herausforderung dar. Üblicherweise wird für schnelles Spiel ein Wechselschlag eingesetzt, da durch das abwechselnde Anschlagen von oben und unten das Tempo, im Gegensatz zu reinen Abschlägen, verdoppelt werden kann. Die Wechselschlagtechnik ist bei Arpeggios oder Melodien mit nur einem Ton pro Saite allerdings weniger effektiv (vgl. Govan 2002b: 34ff.). Für solche Spielarten ist die Technik des Sweepings ${ }^{5}$ besser geeignet, da sich die Anschlagsrichtung nach der Anzahl der Töne pro Saite richtet und unnötige Bewegungen vermieden werden. Im zweiten Takt des Motivs wird eine aufsteigende E-Moll-Skala mit einem abgedämpften Wechselschlag gespielt. Die in den Noten mit P.M. (ausgeschrieben: Palm Mute) gekennzeichnete Technik des Abdämpfens erfolgt mit der Anschlagshand, die während des Spiels

5 Der Begriff »Sweeping « leitet sich vom englischen Verb »to sweep« ab, was so viel wie »fegen « bedeutet. Ähnlich wie mit einem Besen gleitet das Plektrum in einer gleichmäßigen Bewegung und ohne größere Betonungen über die Saiten. 
auf die Saiten nahe am Steg gelegt wird. Daraus resultiert ein kurzer, perkussiver Klang mit einem reduzierten Höhenanteil (vgl. Govan 2003: 25f.). Die geschilderten Spieltechniken gehören zum Standardrepertoire eines fortgeschrittenen Gitarristen und stellen per se keine größere Herausforderung dar. Dies ändert sich jedoch bei einem hohen Spieltempo, das im vorliegenden Beispiel über elf Töne pro Sekunde beträgt und daher besonders aufschlussreich für die Analyse des Einflusses von Verzerrung auf die Spielbarkeit erscheint.

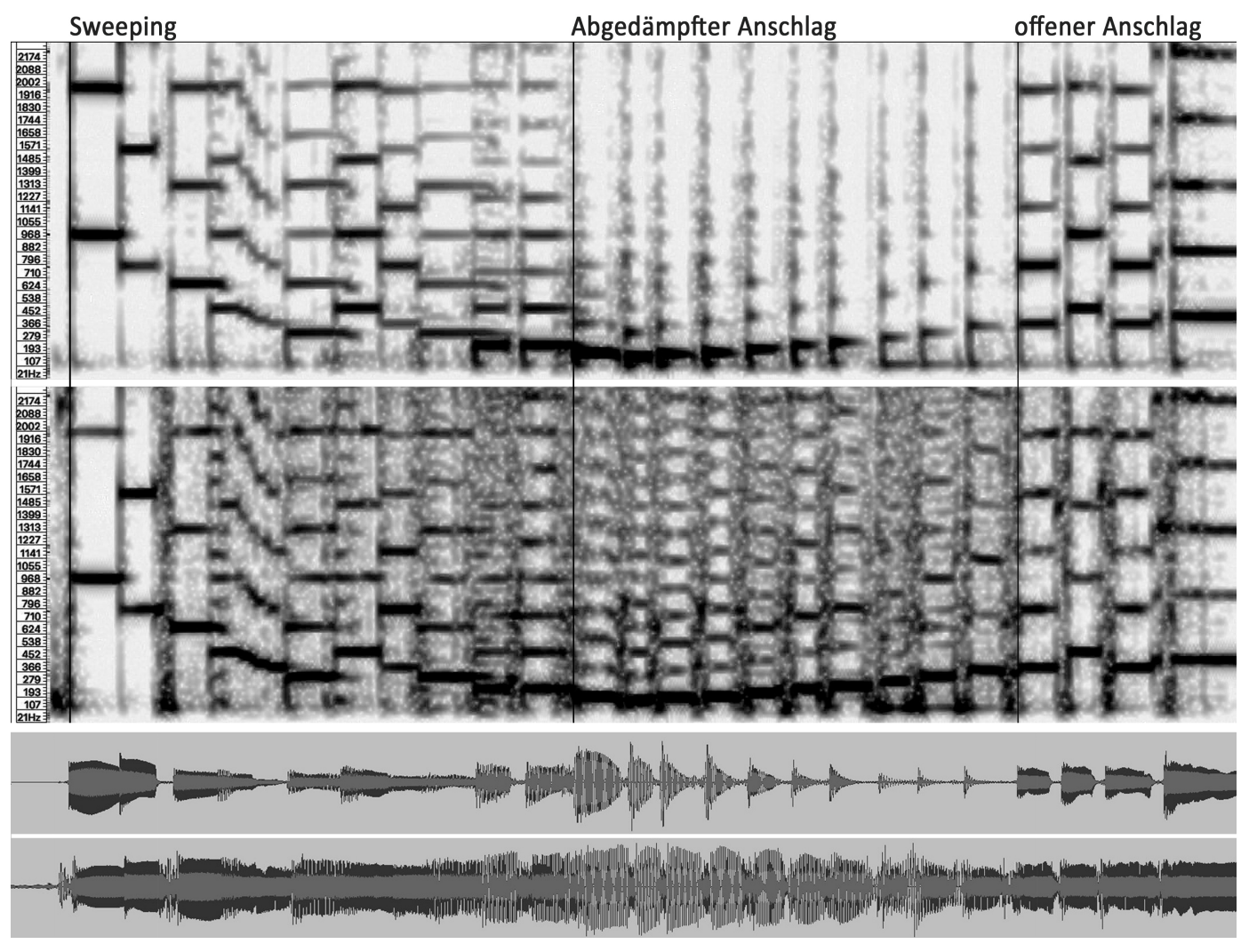

Abbildung 3: Erste zwei Takte von »Groove Or Die« (1997).

Oben: unverzerrt, unten: sehr verzerrt; 21 bis $2.200 \mathrm{~Hz}$

Die Spektral- und Wellenformdarstellungen zeigen charakteristische Unterschiede zwischen abgedämpften (P.M.) und offen gespielten (Wechselschlag und Sweeping) Tönen. In der unverzerrten Aufnahme ist erkennbar, dass sich die freischwingenden Töne - unabhängig der jeweiligen Spieltechnik etwas überlappen, nahtlos ineinander übergehen und daher flüssig klingen. Die abgedämpften Töne haben eine akzentuierte Anschlagsphase und eine kürzere wie leisere Ausklingphase. Dieser Hüllkurvenverlauf zusammen mit der obertonarmen Frequenzverteilung lässt einen wenig transparenten Klang erwarten, und doch ist der Sound dem Höreindruck nach klar artikuliert. Dies ist vermutlich auf die kurzen Pausen zwischen den Tönen zurückzufüh- 
ren, die eine kognitive Differenzierbarkeit der Töne begünstigen. Die verzerrte Gitarre unterscheidet sich bereits auf den ersten Blick durch die größere Anzahl von Teiltönen und deren höheren Lautstärken. Wie die Analyse der Verzerrungscharakteristika bereits nachwies, hat der angeschlagene Ton mit einem verzerrten Sound in allen Phasen der Hüllkurve einen größeren Geräuschanteil (vgl. Einbrodt 1997: 170ff.) - unabhängig von der Anschlagstechnik.

Die Unterschiede zwischen den beiden nicht-abgedämpften Spieltechniken lassen sich in Bezug zum Sound kaum im Spektrogramm finden. Die dynamischen Wellenformdarstellungen verdeutlichen, dass die flüssige Wirkung im unverzerrten Sound durch die verzerrungsbedingte Kompressionswirkung weiter verstärkt wird. Verzerrung verkürzt also die Pausen zwischen den offen angeschlagenen Noten, und beim Sweeping verbindet sie die Töne nahezu übergangslos, was in beiden Fällen zu einem flüssigeren Klangeindruck führt. Bei den abgedämpften Tönen hingegen zeigen sich im Spektrogramm größere Unterschiede zwischen den Sounds. In der verzerrten Aufnahme sind die ersten Obertöne lauter und zusätzlich sind mehrere höhere Teiltöne angedeutet. Aufgrund der Kompression sind die verzerrten Töne in allen Phasen der Hüllkurve laut und haben fließende Übergänge, weshalb abgedämpfte Töne mit zunehmender Verzerrung mehr legato klingen.

Aus Gründen der Daten- und Methodentriangulation wurden die grafischen Analysen mit gemessenen akustischen Werten ausgewählter Töne verglichen. Hierfür wurden die Mittelwerte relevanter Parameter von drei offen gespielten Tönen ( $G 4, B 4, G 4)$, drei abgedämpften Tönen (D4, E4, F\#4) und drei mit Sweeping gespielten Tönen $(E 4, B 3, B 3)$ betrachtet. Hierbei ist zu berücksichtigen, dass die unterschiedlichen Tonhöhen nur eine tendenzielle Vergleichbarkeit der Spieltechniken zuließen:

\begin{tabular}{l|lll|lll} 
& \multicolumn{3}{|c|}{ unverzerrt } & \multicolumn{3}{c}{ verzerrt } \\
& offen & gedämpft & sweeping & offen & gedämpft & sweeping \\
\hline Anschlagsphase & $140 \mathrm{~ms}$ & $80 \mathrm{~ms}$ & $144 \mathrm{~ms}$ & $168 \mathrm{~ms}$ & $146 \mathrm{~ms}$ & $143 \mathrm{~ms}$ \\
Lautheit (RMS) & $-26 \mathrm{~dB}$ & $-24 \mathrm{~dB}$ & $-42 \mathrm{~dB}$ & $-19 \mathrm{~dB}$ & $-19 \mathrm{~dB}$ & $-20 \mathrm{~dB}$
\end{tabular}

Tabelle 2: Akustische Features der Spieltechniken

Die Anschlagsphase wird auf der unverzerrten E-Gitarre zum Teil von der Spielweise beeinflusst. Während sich der offene Anschlag und das Sweeping nur unwesentlich unterscheiden, verfügt die abgedämpfte Spieltechnik erwartungsgemäß über eine deutlich kürzere Anschlagsphase. Bei einem verzerrten Sound hingegen ist die Anschlagsphase der abgedämpften Töne nur etwas kürzer. Ähnliche Phänomene zeigen sich bezüglich der Lautheit. Wäh- 
rend auf der unverzerrten Gitarre die mit Sweeping gespielten Töne deutlich leiser sind, ist die Intensität mit einem verzerrten Sound bei allen Spieltechniken fast identisch. Unter Berücksichtigung der grafischen und akustischen Ergebnisse kann gefolgert werden, dass die Unterschiede zwischen den Spieltechniken beim unverzerrten Sound größer sind. Verzerrung scheint zu Angleichungen zu führen, weil sie die klanglichen Eigenschaften verschiedener Spielweisen reduziert. Dies verdeutlicht auch ein Frequenzvergleich eines G3-Tons, der in dem Sampleausschnitt sowohl offen als auch abgedämpft erklingt:

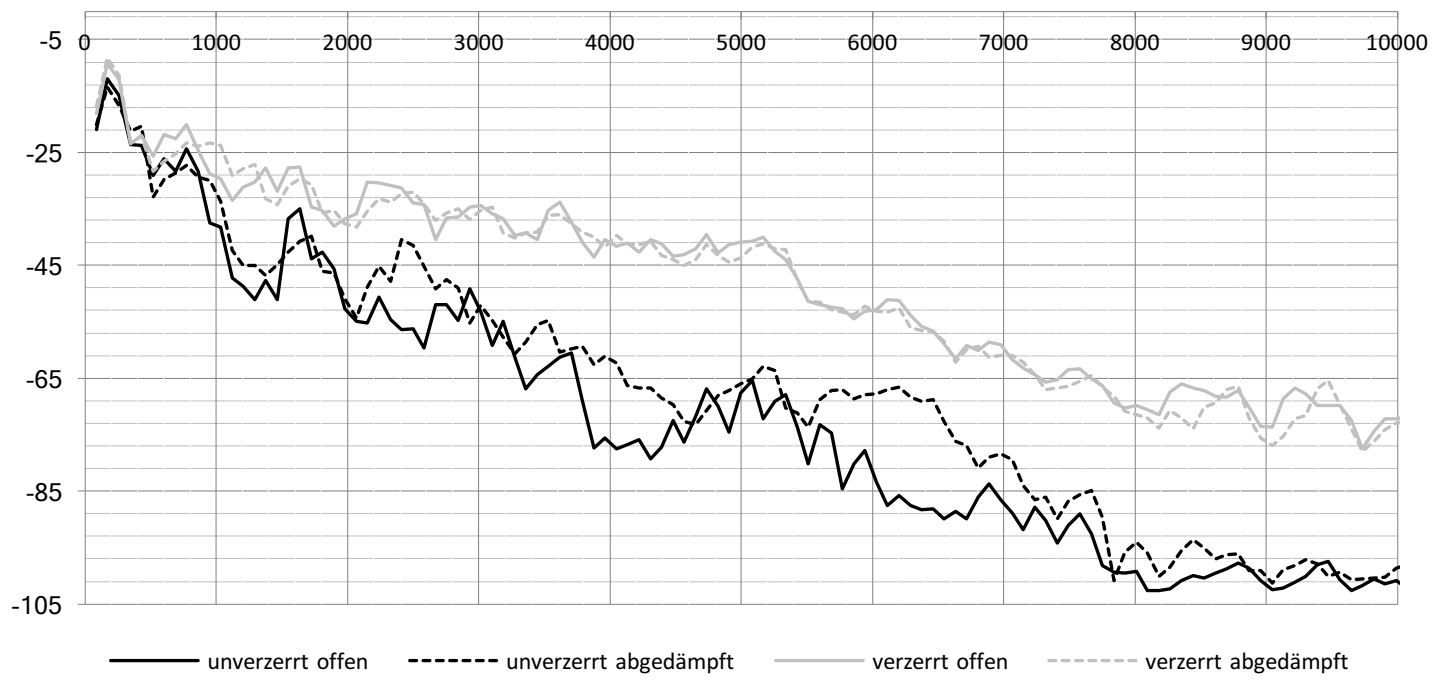

Abbildung 4: Frequenzanalyse des G3-Tons unterschieden nach Spielweise und Verzerrungsgrad

Bezüglich Virtuosität stellt sich nun die Frage, wie der Einfluss von Verzerrung auf das Spiel zu bewerten ist. Zunächst kann festgehalten werden, dass Verzerrung die Töne ungeachtet der jeweiligen Spieltechnik verlängert und damit einen flüssigeren Klangeindruck bewirkt. Wie die vorangegangene akustische Analyse bereits nahelegte, verlängert Verzerrung die Anschlagszeit und -phase, was zusammen mit dem erhöhten Geräuschanteil zu einem weniger artikulierten Anschlag führt. Diese Beobachtung stimmt mit den spektralen und dynamischen Ergebnissen überein.

In der Praxis gilt, dass ein sauberes Gitarrenspiel die präzise Abstimmung zwischen Greif- und Anschlagshand verlangt, damit der Ton nicht abgehackt und undefiniert klingt (vgl. Weissberg 2010: 99f.). Die akustischen Klangeigenschaften der Verzerrung können solche Unsauberkeiten zu einem gewissen Grad maskieren, sodass mangelnde Präzision weniger auffällt und der ausführende Interpret schneller als mit einem unverzerrten Sound spielen und dem Hörer bzw. Zuschauer eine höherentwickelte Spielfähigkeit 
»vorgaukeln« kann. Vor allem Nicht-Gitarristen und Gitarrenanfänger können eine nur schnelle Spielweise schwerlich von einem wirklich virtuosen Spiel unterschieden. Abgesehen von den geringeren Erfordernissen einer synchronen Handabstimmung werden durch die Kompression ungleichmäßige Lautstärken wie auch fehlende Dynamik und Akzente kompensiert.

Insgesamt betrachtet können die akustischen Auswirkungen der Verzerrung das expressive Potenzial der Gitarre gleichermaßen erweitern wie auch beschränken. Im ungünstigen Fall beeinträchtigt Verzerrung die Klarheit in schnellen Melodielinien. Diese negative Folge kann durch abgedämpftes Spiel mit kleinen Pausen zwischen den Tönen gemindert werden, ohne dass die flüssige Wirkung verloren geht. Je nach Präzision erzeugt Verzerrung in Verbindung mit einem abgedämpften Anschlag einen präzisen und kraftvollen Sound; oder aber unsauberes Spiel bzw. Abstimmungsprobleme zwischen den Händen werden kaschiert. Durch das Abdämpfen werden die Grundtonfrequenz und die ersten Teiltöne verstärkt, sodass ein voller und definierter Sound entsteht. Ein positiver Nebeneffekt ist die Reduzierung störender Geräusche von nicht gespielten Saiten. Zu viel Verzerrung hingegen maskiert die Töne durch Geräusche (vgl. Einbrodt 1997: 170ff.), sodass selbst sauberes Spiel an Klarheit verliert. Ferner minimiert Verzerrung die dynamischen Ausdrucksmöglichkeiten, was die Expressivität des Spiels einschränken kann.

\section{Legato}

Bei der Gitarre beruhen Legato-Techniken auf dem weitgehenden Verzicht auf Plektrumanschläge, deren Akzentuierung den flüssigen Klang unterbrechen. Die Legato-Spielweisen werden wegen ihrer Klangwirkung, aber auch aus spieltechnischen Gründen verwendet, wie die Forenanalyse verdeutlichte. Die Auswirkung von Verzerrung auf das Spiel wird an den ersten zwei Takten des Introsolos von Queens »I Want It All« (1989) untersucht:

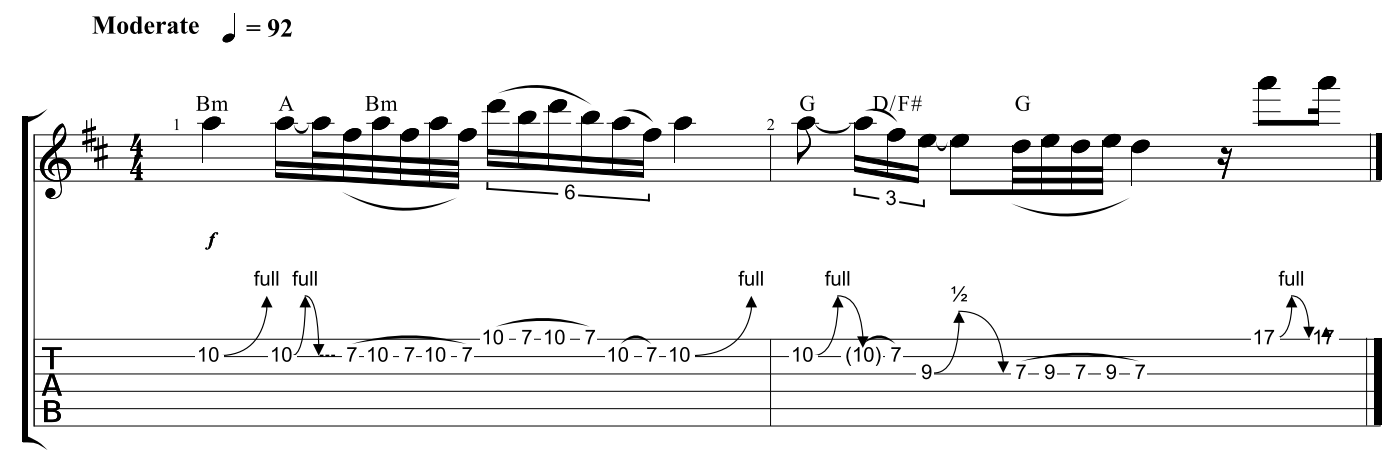

Abbildung 5: Anfang des Introsolos von Queens »I Want It All« (1989) 
Beide experimentellen Aufnahmen verdeutlichen im Spektrogramm eine weitgehend geräuschfreie Einschwing- wie Ausschwingphase legato gespielter Töne. Die gezogenen Töne (»Bendings«) sind angeschlagen und verfügen über ein lauteres und länger anhaltendes Geräusch. Diese unharmonischen Klanganteile lassen sich auch in den Legato-Phrasen erkennen, da angeschlagene Töne aufgrund von Saitenwechseln vorkommen. Aus dem Vergleich der beiden Tonerzeugungsweisen kann geschlossen werden, dass der Legato-Klangeindruck auf eine geringere Geräuschhaftigkeit zurückzuführen ist. Dies bekräftigt die Klanganalyse, denn die Anschlagsphase verlängert sich im Legato-Spiel durch ein Hinzufügen von Verzerrung nur um $10 \%$ gegenüber einer Verlängerung um $28 \%$ bei angeschlagenen Tönen. Die verkürzte Anschlagsphase scheint folglich ein Merkmal des Legato-Klanges zu sein.

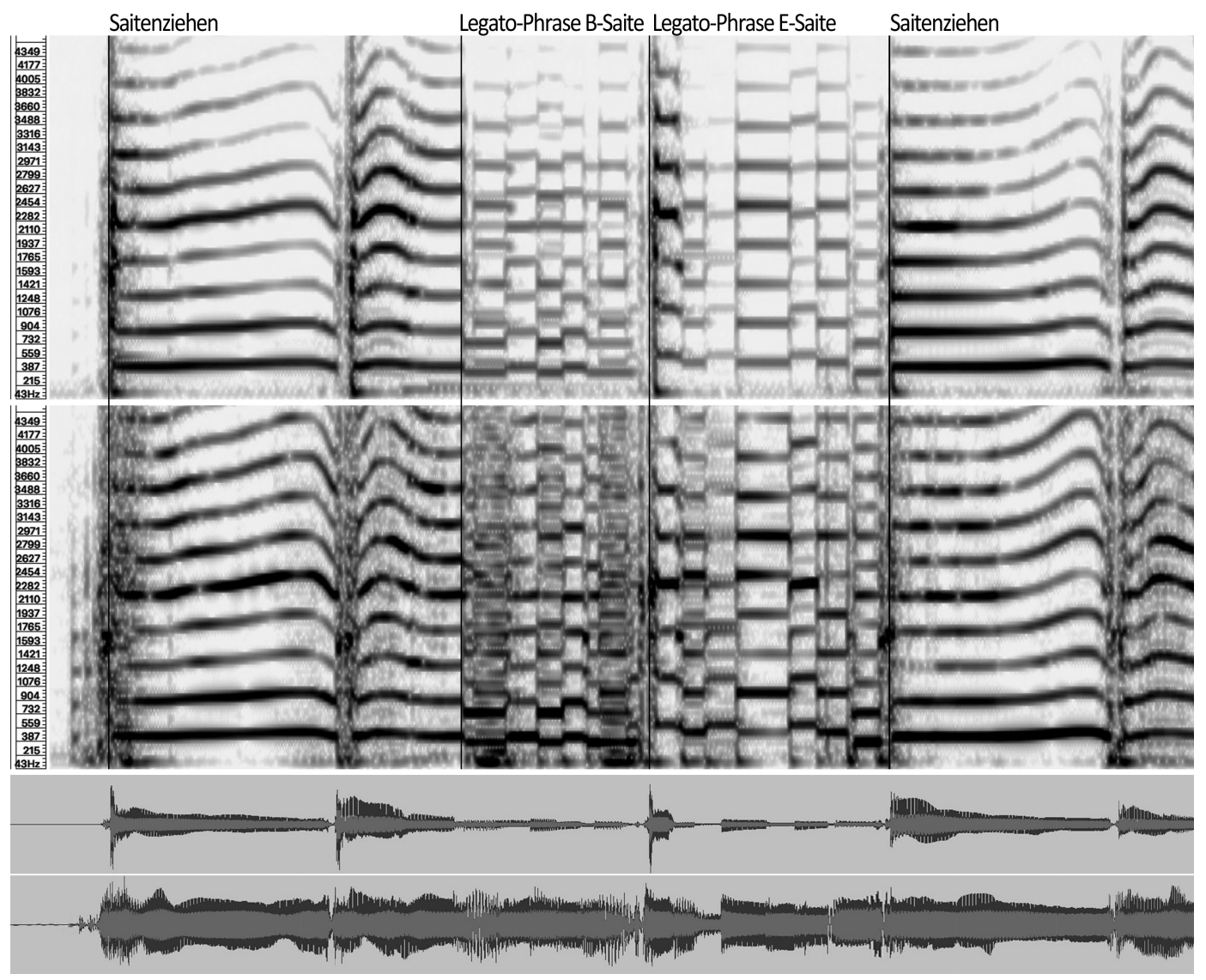

Abbildung 6: Anfang des Introsolos von Queens »I Want It All« (1989).

Oben: unverzerrt, unten: sehr verzerrt; 43 bis $4.350 \mathrm{~Hz}$

Beide grafischen Darstellungen liefern Anzeichen für eine vereinfachte Spielbarkeit der Legato-Technik durch einen verzerrten Sound. In der unverzerrten Aufnahme sind die Teiltöne der angeschlagenen Töne wesentlich lauter als diejenigen der legato gespielten Töne. Im Höreindruck sind die 
gebundenen Töne deutlich leiser, sodass sie in einer Bandsituation leicht untergehen würden. Beim verzerrten Sound zeigt sich die Kompression darin, dass alle Teiltöne unabhängig von der jeweiligen Spieltechnik verstärkt sind. Noch eindeutiger unterstützt die Wellenformdarstellung diese Beobachtung, denn Verzerrung wandelt den perkussiven Klangeindruck des unverstärkten Instruments in ein lang ausklingendes Instrument, unabhängig der Spieltechnik. Die Feature-Analyse bestätigt die unterschiedliche Dynamikrelation. Während das gebundene Spiel auf der unverzerrten Gitarre durchschnittlich 13,29 dB leiser als das angeschlagene ist, beträgt die Differenz mit einem verzerrten Sound lediglich 4,61 dB. Diese eingeschränkte Dynamik erleichtert es dem Spieler, beide Techniken in einer Phrase zu benutzen. Zwar ist eine ähnliche Lautstärke durch Übung zu erreichen, allerdings wird die exakte Ausführung bei höheren Spielgeschwindigkeiten schwieriger, wobei Verzerrung hilft, das Spiel durch Kompression zu vereinfachen und ähnlich wie beim Plektrumspiel spieltechnische Mängel zu maskieren. Insbesondere ein zweihändiges Legato-Spiel (»Tapping«) wird durch Verzerrung deutlich leichter, da weniger Kraftaufwand erforderlich ist. Darüber hinaus verdeutlichen die grafischen Analysen und die akustischen Werte, dass Verzerrung die Dauer der Töne verlängert. Nicht nur schwingen die Teiltöne aufgrund der Kompression länger aus, auch setzt der Ton früher ein, was die Töne mit weniger oder kürzeren Pausen verbindet. Mit einer unverzerrten oder akustischen Gitarre braucht es deutlich mehr Anstrengung, um einen solch flüssigen Klang zu erzielen.

\section{Diskussion und Fazit}

Der Beitrag untersuchte die Auswirkungen von Verzerrung auf die Spielbarkeit der E-Gitarre unter besonderer Berücksichtigung des »Shreddings«, dem schnellen Solospiel. Während wissenschaftliche Studien (vgl. Walser 1993: 57ff.; Weissberg 2010: 99f.) die begünstigenden Effekte dieses Sounds auf ein virtuoses Solospiel im Rock und Metal bislang nur vermuteten, zeigte die Inhaltsanalyse der Musikerforen viele Facetten des verzerrten Gitarrenspiels; sie betonte aber auch die vereinfachende Wirkung. Die seltenen Ausführungen zur Verzerrung in der instrumentalpädagogischen Literatur, die an dieser Stelle nicht näher beschrieben werden können (vgl. Herbst 2016: 239-255), beschränken sich ebenfalls weitgehend auf die Vereinfachung, weshalb mehrheitlich das Üben mit einem unverzerrten Sound empfohlen wird (vgl. Culpepper 1996: 2; Govan 2003: 18). 
Die Ergebnisse der Fallanalysen liefern akustische Belege für die spielvereinfachende Wirkung der Verzerrung, hauptsächlich aufgrund von Kompression, mehr Sustain sowie der veränderten Hüllkurve. Viele Spieltechniken wie Wechselschlag, Sweeping, künstliche Obertöne, Hammer-On, PullOff und Zweihand-Tapping profitieren von den akustischen Veränderungen, andere hingegen, etwa ein kontrolliertes Feedback, hängen sogar davon ab (vgl. Herbst 2016: 143-237). Da es vor allem beim Wechselschlag als der Standardtechnik des Melodiespiels schwer ist, die Hände beim »Shredding « zu synchronisieren, verhilft Verzerrung dem Spiel zu einem flüssigeren und gleichmäßigeren Klangeindruck. Die Töne verbinden sich, Lautstärkeunterschiede und Akzente werden reduziert und überdies findet eine Angleichung der Spieltechniken statt, sodass mangelnde oder fehlerhafte Phrasierungsfähigkeiten weniger auffallen. Einige dieser Folgen der Verzerrung wurden von den Gitarristen in den Foren kritisch diskutiert.

Selbst wenn den Ergebnissen zufolge das schnelle Spiel durch Verzerrung begünstigt wird, dürfen die in den Foren ebenfalls genannten Herausforderungen nicht außer Acht gelassen werden. Einige der Schwierigkeiten werden auch in den wenigen instrumentalpädagogischen Texten betont:

»a heavily overdriven amp is a frisky beast, and the slightest of accidental hand movements at your end can turn into an enormous and unpleasant racket by the time it reaches the speakers. In a context like this, you have to be more conscious than ever of damping all unwanted strings, just to make sure that there's a difference in volume level between when you're playing and when you're not« (Govan 2003: 26).

Zusätzlich zum Verstärkerrauschen erzeugt die Kompression einen intensiven Geräuschuntergrund, wodurch selbst kleinste Schwingungen von nicht gespielten Saiten im Gesamtklang auffallen (vgl. Einbrodt 1997: 175) und sogar die gespielten Töne überdecken können. Vor allem kurze Spielpausen aber auch gegriffene Töne können durch Rückkoppelungen zum Problem werden (vgl. Govan 2003: 19). Rock- und Metal-Gitarristen müssen deshalb zu Experten der klanglichen Kontrolle werden, damit das Gespielte klar bleibt und Nebengeräusche nicht im Bandzusammenklang auffallen (vgl. ebd.: 18). Einerseits müssen nicht gespielte Saiten gedämpft werden. Das geschieht vor allem mit dem Zeigefinger der Greifhand, der durch leichte Berührung diese Saiten am Schwingen hindert. Andererseits muss die Anschlagshand die Schwingung von tieferen Saiten unterdrücken, während die höheren Saiten gespielt werden (vgl. Baxter 2002: 65f.). Die Ausführung erfordert eine gute Synchronisation mit der Greifhand (vgl. Govan 2003: 26) und einen kontrollierten Druck auf die entsprechenden Saiten am Steg, da- 
mit die gespielten Töne nicht versehentlich abgedämpft werden (vgl. Baxter 2002: 65).

Eine andere Konsequenz der Verzerrung, die in den Forenbeiträgen kaum zum Ausdruck kam, ist die eingeschränkte Dynamik, die sich negativ auf die Ausdrucksfähigkeit auswirken kann. Das Spiel mit einem verzerrten Sound erfordert mehr Aufwand, um Akzente und Phrasen dynamisch zu gestalten. Hinzu kommt, dass ein Crescendo in Abhängigkeit vom Verzerrungsgrad weniger die Lautstärke erhöht, sondern eher die Klangfarbe verändert (vgl. Govan 2003: 29). Ein kräftigerer Anschlag führt zu stärkerer Verzerrung mit mehr Obertönen und Geräuschanteilen. Dahingegen produziert ein sanftes Anschlagen einen wärmeren Klang (vgl. Einbrodt 1997: 170ff. und 210f.). Folglich muss der Verzerrungsgrad während des Spiels mit dem Lautstärkeregler des Instruments flexibel eingestellt werden, um ein größeres Dynamik- und Klangspektrum zu erreichen. Das Beherrschen solcher Fertigkeiten unterscheidet vermutlich den »wahren« Virtuosen vom »nur schnellen« Spieler (vgl. Reimer 1972: 572f.).

Das Abdämpfen (»Palm Mute«) ist eine wichtige Artikulationstechnik für ein dynamisches Spiel. Es verringert den charakteristischen flüssigen Klangeindruck der Verzerrung, ohne die Wirkung der Kompression zu reduzieren. Ein kraftvoller perkussiver Klang resultiert daraus, der je nach Druck und Position der Hand höhen- oder bassbetonter ist (vgl. Govan 2003: 25). Allerdings muss der Spieler zur Vermeidung unsauberer Intonation gleichzeitig darauf achten, die Tonhöhe nicht zu verändern (vgl. ebd.). In schnellen Sololäufen kann die bewusste Auswahl abgedämpfter und offen gespielter Töne zur Betonung einzelner Melodietöne genutzt werden, was jedoch viel Kontrolle erfordert. Bevorzugt wird die Dämpftechnik auf den tiefen Saiten angewandt, da dies die klare Trennung der tiefen Töne beim schnellen Spiel begünstigt (vgl. Herbst 2016: 243). Die Glättungswirkung der Verzerrung wird reduziert, was ein synchroneres Spiel beider Hände sowie die Klanggestaltung durch die Anschlagshand notwendig macht. Diese Vorgehensweise ist weniger ein Zeichen besonderer Virtuosität, sondern vielmehr ein Erfordernis für einen differenzierten Sound. Dennoch ist auch nicht-abgedämpftes Spiel nicht einseitig mit vereinfachtem Spiel gleichzusetzen, da die verzerrungsbedingte Betonung des Höhenbereichs die Klarheit der Artikulation verbessert und ein nicht-synchrones Spiel beider Hände auffallen würde (vgl. Govan 2003: 20).

Ein weiterer Unterschied zwischen den Sounds ist in der verlängerten Tondauer der verzerrten Gitarre begründet. Dieses Phänomen kann zwar eine mangelnde Synchronisierung zwischen beiden Händen maskieren; einerseits ist es aber schwieriger, die Töne rhythmisch genau abzustoppen, 
und andererseits führen überlappende Töne nicht nur zu wenig angenehmen Schwebungen, sondern reduzieren auch die Klarheit der Melodie. Beim Sweeping ist dies besonders problematisch (vgl. Govan 2002a: 63), wie es einige Aussagen in den Foren bestätigen.

Die Verzerrung kann in mancherlei Hinsicht die Expressivität einschränken oder zumindest die Phrasierung erschweren, dennoch muss ihr zugestanden werden, dass sie viele gitarrenspezifische Spiel- und Ausdrucksweisen in den Genres Blues, Rock und Metal geprägt hat (vgl. Waksman 2003a: 114f.; Herbst 2016: 143-237). Nach Walser (1993: 42) und Jauk (2009: 268f.) nähert sich die verzerrte Gitarre der Gesangsstimme an, indem die Klangfarbe eine Ähnlichkeit mit einem rauen Stimmtimbre erhält. Ebenso erweitern sich bestimmte Phrasierungsmöglichkeiten. Bspw. kommen das Saitenziehen (»Bending «) wie auch intensivere Formen des Vibratos durch das verlängerte »Sustain« besser zur Geltung (vgl. Herbst 2016: 178ff.). Die Wahl des Tonabnehmers und die Anschlagsposition ermöglichen es außerdem, verschiedene Vokalklänge in Abstimmung mit dem Volumenregler zu imitieren (vgl. Traube/Depalle 2004). Die Spieltechnik kombiniert mit der Soundgestaltung am Instrument bewirkt eine Klangfilterung wie ein Equalizer (vgl. Jauk 2009: 268). Dieser Effekt kann durch ein Wah Wah-Pedal noch intensiviert werden, wenngleich auch diese Art der Klanggestaltung sensibel auf den Grad der Verzerrung reagiert (vgl. Herbst 2016: 181ff.). Selbst das Gesangsfalsett kann durch künstliche Obertöne und Feedback imitiert werden (vgl. ebd.: 178ff.). So folgert Gitarrenvirtuose Christopher Amott, dass sich das unverzerrte deutlich von einem verzerrten Gitarrenspiel unterscheidet:

»distortion is almost an instrument in itself [...]. There are many beautiful tones and effects you can get from it, as all the little details of your playing, like pick scrapes and overtones come through stronger than if you were to play with a clean sound « (zit. n. ebd. 2016: 321).

Nach vorausgegangener Betrachtung kann resümiert werden, dass die Vorstellung von Verzerrung als eine rein vereinfachende und somit potenziell »betrügende « Klanggestaltung im Sinne des virtuosen »Shreddings « als zu kurz gegriffen scheint. Diese Erkenntnis wird in den regen Forumsdiskussionen untermauert, wo die Verzerrungsbefürworter stets die Herausforderungen betonen, wenngleich sie mehr die Nebengeräusche thematisieren als die Schwierigkeiten einer artikulierten Phrasierung zu benennen. Vor allem Anfänger sehen sich mit dem Problem konfrontiert, verzerrungsbedingte Nebengeräusche zu kontrollieren. Darin stimmen die musik- und inhaltsanalytischen Ergebnisse überein. Von Verzerrung profitieren vor allem fortge- 
schrittene Spieler, denn die Auswirkungen dieses Sounds erlauben insbesondere in der »Shred-Spielweise « ein schnelleres, aber nicht zwangsläufig ein saubereres Spiel. Ein hohes Maß an Kontrolle wird jedoch benötigt, um die teilweise eingeschränkten Ausdrucksmöglichkeiten auszugleichen und detaillierte Klangnuancen phrasieren zu können (vgl. Govan 2003: 18ff.). Daher ist es auch entgegen vieler Ratschläge (vgl. Culpepper 1996: 2) sinnvoll, ja gar notwendig, mit und ohne Verzerrung zu üben, um sowohl die saubere Ausführung sicherzustellen als auch das expressive Potenzial dieses Sounds bestmöglich zu nutzen. »Wahre Virtuosen wie bspw. Eddie Van Halen, Paul Gilbert, Steve Morse, John Petrucci, Joe Satriani oder Steve Vai sind sich der Vielzahl an Gestaltungsmöglichkeiten bewusst und können diese gezielt in schnellen wie in langsamen Phrasen einsetzen. Folglich sollten die vielfältigen gestalterischen Möglichkeiten, die Verzerrung bietet, von »ShredGitarristen« erkannt werden. Daher wäre es wünschenswert, wenn sich der Fokus vom rein negativen Image des »Cheatings « zum expressiven Potenzial hin verlagern würde. Virtuoses E-Gitarrenspiel erfordert ungeachtet des Verzerrungsgrads einerseits die volle Kontrolle über das Instrument. Gleichzeitig müssen andererseits die speziellen Ausdrucksweisen mit verschiedenen Sounds für die Umsetzung der musikalischen Intention beherrscht werden.

\section{Literatur}

Barkowsky, Johannes (2009). Einführung in die musikalische Akustik. Wilhelmshaven: Florian Noetzel.

Baxter, Shaun (2002). »The Guitar Gym. Alternate Picking. «In: Guitar Techniques 6, S. 64-68.

Cook, Nicholas / Clarke, Eric (2004). »Introduction: What Is Empirical Musicology?« In: Empirical Musicology. Aims, Methods, Prospects. Hg. v. Nicholas Cook und Eric Clarke. Oxford: Oxford University Press, S. 3-14.

Culpepper, Carl (1996). Terrifying Technique For Guitar. The Ultimate Source For Building Chops. Milwaukee, WI: Hal Leonard.

Custodis, Michael (2011). Living History. The Guitar Virtuoso and Composer Steve Vai. Online-Publication Münster, http://www.uni-muenster.de/imperia/md/ content/musikwissenschaft/pdf/custodis-vai.pdf (Stand vom 23.3.2017).

Doyle, Mike (1993). The Sound of Rock. A History of Marshall Valve Guitar Amplifiers. Westport, CT: The Bold Strummer.

Einbrodt, Ulrich Dieter (1997). Experimentelle Untersuchungen zum Gitarrensound in der Rockmusik. Frankfurt/M.: Peter Lang.

Elflein, Dietmar (2010). Schwermetallanalysen: Die musikalische Sprache des Heavy Metal (= texte zur populären musik 6). Bielefeld: transcript. 
Frudua, Galeazzo (2010). »Advantages and Disadvantages of Scalloped Fretboards.« In: The Frudua Guitar Craftpedia, http://www.frudua.com/scalloped_ fretboards.htm (Stand vom 23.3.2017).

Govan, Guthrie (2002a). "11 Red Hot Metal Licks.« In: Guitar Techniques 4, S. 58-63.

Govan, Guthrie (2002b). Creative Guitar 2: Advanced Techniques. London: Sanctuary.

Govan, Guthrie (2003). Creative Guitar 1: Cutting Edge Techniques. London: Sanctuary.

Herbst, Jan-Peter (2016). Die Gitarrenverzerrung in der Rockmusik. Studien zu Spielweise und Ästhetik. Münster: LIT.

Jauk, Werner (2009). Popmusic + MedienKunst: Der musikalisierte Alltag der digital culture. Osnabrück: epOs Music.

Kuckartz, Udo (2010). Einführung in die computergestützte Analyse qualitativer Daten. Wiesbaden: Springer.

Lartillot, Olivier / Toiviainen, Petri (2007). »A Matlab Toolbox for Musical Feature Extraction From Audio. « In: Proceedings of the $10^{\text {th }}$ International Conference on Digital Audio Effects. Bordeaux, http://dafx.labri.fr/main/papers/p237.pdf (Stand vom 23.3.2017).

McAdams, Steve / Depalle, Phillippe / Clarke, Eric (2004). »Analyzing Musical Sound.« In: Empirical Musicology. Aims. Methods. Prospects. Hg. v. Eric Clarke und Nicholas Cook. Oxford: Oxford University Press, S. 157-196.

Mclver, Joel (2008). The 100 Greatest Metal Guitarists. London: Jawbone.

Metzner, Paul (1998). Crescendo of the Virtuoso. Spectacle, Skill, and Self-Promotion in Paris during the Age of Revolution. Berkeley, CA: University of California Press.

Middleton, Richard (1990). Studying Popular Music. Buckingham: Open University Press.

Obrecht, Jay (2010). »Randy Rhoads: The 1982 Max Norman Interview.« In: Jas Obrecht Music Archive, http://jasobrecht.com/randy-rhoads-max-norman-interview (Stand vom 23.3.2017).

Pierce, John R. (1985). Klang. Musik mit den Ohren der Physik. Heidelberg: Spektrum.

Pincherle, Marc (1949). »Virtuosity.«In: The Musical Quarterly 35, Nr. 2, S. 226243.

Reimer, Erich (1972). »Virtuose.«In: Handwörterbuch der musikalischen Terminologie. Hg. v. Hans Heinrich Eggebrecht. Stuttgart: Steiner, S. 569-576.

Schneider, Albrecht (2002). "Klanganalyse als Methodik der Popularmusikforschung. «In: Musikwissenschaft und populäre Musik. Versuch einer Bestandsaufnahme. Hg. v. Helmut Rösing, Albrecht Schneider und Martin Pfleiderer (= Hamburger Jahrbuch für Musikwissenschaft 19). Frankfurt/M.: Peter Lang, S. 107-127.

Slaven, James E. / Krout, Jody L. (2016). »Musicological Analysis of Guitar Solos from the Roots of Rock through Modern Heavy Metal «. In: Metal Music Studies 2, Nr. 2, S. 245-251.

Traube, Caroline / Depalle, Phillippe (2004). »Phonetic Gestures Underlying Guitar Timbre Description. "In: Proceedings of the 8th International Conference of Music Perception and Cognition (ICMPC 8). Evanston, IL, https://www. researchgate.net/publication/228712315_Phonetic_gestures_underlying_guitar_ timbre_description (Stand vom 23.3.2017). 
Waksman, Steve (2001). »Into the Arena: Edward Van Halen and the Cultural Contradictions of the Guitar Hero. «In: Guitar Cultures. Hg. v. Andy Bennett und Kevin Dawe. Oxford: Berg, S. 117-134.

Waksman, Steve (2003a). »The Turn to Noise: Rock Guitar from the 1950s to the 1970s. « In: The Cambridge Companion to the Guitar. Hg. v. Victor Anand Coelho. Cambridge, MA: Cambridge University Press, S. 109-121.

Waksman, Steve (2003b). »Contesting Virtuosity: Rock Guitar since 1976.«In: The Cambridge Companion to the Guitar. Hg. v. Victor Anand Coelho. Cambridge, MA: Cambridge University Press, S. 122-132.

Walser, Robert (1993). Running with the Devil. Power, Gender, and Madness in Heavy Metal Music. Hanover, NH: Wesleyan University Press.

Weissberg, Daniel (2010). »Zur Geschichte elektroakustischer Instrumente aus dem Blickwinkel der Körperlichkeit.« In: Klang (ohne) Körper. Spuren und Potenziale des Körpers in der elektronischen Musik. Hg. v. Michael Harenberg und Daniel Weissberg. Bielefeld: transcript, S. 91-104.

\begin{abstract}
"Shredding «, the fast and virtuous guitar playing, is a central stylistic element of many rock and metal music genres. Previous research has claimed distortion to increase the guitar's potential as a virtuoso solo instrument but acoustic evidence is still missing. Apart from this academic desideratum, distortion is lively debated in online musicians' boards for its supposed »cheating « effect. This study aims at investigating distortion's effect on the electric guitar tone and its consequences for virtuoso playing. After a qualitative content analysis of discussions in online boards, an analytic case study of two guitar solos examines distortion's effect on musical performance. The findings confirm distortion's simplifying effect whilst also highlighting challenges less commonly considered in research, journalism, and performance practice.
\end{abstract}

